\title{
UTILIZAÇÃO DE FORMIGAS (HYMENOPTERA: FORMICIDAE) COMO BIOINDICADORAS EM PLANTIOS DE PINUS NO PARANÁ
}

\author{
Karen Koch Fernandes de Souza ${ }^{1}$, Nilton José Sousa², Ivan Crespo Silva², Pedro Pacheco dos Santos \\ Lima $^{3}$, Eli Nunes Marques ${ }^{4}$
}

\begin{abstract}
RESUMO - Os cultivos florestais transformam a paisagem e, como as demais monoculturas, apresentam baixa diversidade, sendo que a qualidade dos plantios pode ser investigada por meio da utilização de bioindicadores. As formigas são amplamente empregadas como indicadores edáficos porque compreendem um grupo numericamente abundante, possuem ampla diversidade, ocupam diferentes nichos e, comparadas a outros seres vivos, são fáceis de amostrar, além de responderem as pressões ambientais. Esta pesquisa procurou realizar levantamento qualitativo e quantitativo das espécies de formigas de solo presentes em quatro ambientes (remanescente de Floresta Ombrófila Mista e três idades de plantios de Pinus sp.). As coletas ocorreram em quatro etapas distribuídas ao longo de um ano. Em cada unidade amostral foi instalado um transecto de armadilha do tipo pitfall (adaptadas para uso com iscas de sardinha em óleo comestível) contendo cinco armadilhas distanciadas $10 \mathrm{~m}$ entre si. No total, foram coletados 3374 indivíduos pertencentes à Família Formicidae distribuídos em 23 espécies. A análise qualitativa mostrou que o gênero Pheidole teve o maior registro de indivíduos. O gênero Solenopsis foi o mais frequente no plantio de Pinus taeda aos oito anos de idade, revelando que o gênero pode estar associado a ambientes perturbados enquanto que o gênero Pachycondyla sp. foi observado com maior frequência em ambiente com melhor estado de conservação.
\end{abstract}

Palavras chave: mirmecofauna, bioindicadores, Pinus sp.

\section{UTILIZATION OF ANTS (HYMENOPTERA: FORMICIDAE) AS BIOINDICATORS IN PINUS PLANTING IN PARANÁ STATE, BRAZIL}

\begin{abstract}
Forest crops transform the landscape and, like other monocultures, have low diversity. The quality of the crops can be ascertained by using bioindicators. Ants are widely used as indicators because edaphic comprise a numerically abundant group, have extensive diversity, occupy different niches and, compared to other living beings, are easy to sample, and respond to environmental pressures. This research sought to carry out qualitative and quantitative survey of the species of soil ants present in four environments (remnant of Araucaria Forest and three ages of Pinus sp. plantations). The samples were collected in four stages distributed throughout the year. In each sample unit were installed one trap transect pitfall type (adapted for use with sardine baits in edible oil) containing five traps $10 \mathrm{~m}$ away. In total, we collected 3374 individuals belonging to the family Formicidae distributed in 23 species. Qualitative analysis showed that Pheidole gender had the greatest individual record. The Solenopsis genus was the most frequent in planting loblolly pine to eight years old, showing that the genre may be associated with disturbed environments while gender Pachycondyla sp. It was observed more frequently in an environment with better condition.
\end{abstract}

Keywords: ants, bioindicators, Pinus sp.

\footnotetext{
${ }^{1}$ Programa de Pós-graduação em Engenharia Florestal da Universidade Federal do Paraná - UFPR, Professora da Pontifícia Universidade Católica do Paraná - PUCPR, Curitiba, Paraná (Autor para correspondência - email: karenkfs@yahoo.com.br)

${ }^{2}$ Professores do Departamento de Ciências Florestais - UFPR, Universidade Federal do Paraná - UFPR

${ }^{3}$ Especialista da Universidade de São Paulo, Faculdade de Medicina Veterinária e Zootecnia, Campus Pirassununga, SP.

${ }^{4}$ Professor sênior da Universidade Federal do Paraná, UFPR.
} 


\section{INTRODUÇÃO}

Os plantios florestais são essenciais para a sociedade, fornecem produtos diretos (madeira, celulose, resina, frutos, fibras, entre outros) além de serem reconhecidos pelos serviços ambientais - conservação de recursos hídricos e edáficos, sequestro de carbono, entre outros. No entanto, por se tratar de monocultura, simplificam o ambiente e alteram as condições naturais do meio, sendo possível avaliar o impacto causado pelo cultivo florestal por meio da amostragem de indicadores ambientais.

Entre as principais espécies cultivadas, o gênero Pinus se destaca no sul e sudeste do país, por se ajustar as características edafoclimáticas. De acordo com Medrado (2008) as plantações de pinus tem uma grande importância para o setor florestal brasileiro, pois agregaram valor desde o pequeno produtor rural até os vários segmentos industriais como laminação, serraria, papel e celulose, chapas e geração de energia.

Os formicídeos se constituem em um importante grupo para o monitoramento ambiental (Oliveira et al., 2014). As formigas são insetos sociais que vivem em colônias formadas por castas (Borror \& Delong, 1969), podendo ser encontradas em uma variedade de locais, desde desertos a florestas úmidas (Kaspari, 2000).

Entre os atributos que tornam as formigas ideais para a serem utilizadas como bioindicadores, Alonso \& Agosti (2000) destacam a presença em diversos habitats e distribuição em todo o mundo, a alta diversidade, são numericamente abundantes, são relativamente fáceis de coletar, respondem às mudanças ambientais, e, ainda, possuem hábitos de nidificação estacionário que permitem a reamostragem ao longo do tempo.

$\mathrm{O}$ uso de formigas como indicadores, tem sido objeto de estudo, em diversos trabalhos na Austrália, especialmente, em estudos de reabilitação de áreas degradadas oriundas da atividade de mineração (Majer, 1983; Majer \& Nichols, 1998). A investigação acerca do tema no Brasil pode ser observada em trabalhos relacionados à fragmentação florestal (Vasconcelos, 1998; Liberal et al., 2007; Oliveira et al., 2016; Silva et al., 2018), a agroecossistemas (Dias, 2004; Spolidoro, 2009; Cantarelli et al., 2015) e também em áreas degradadas pela mineração (Diehl-Fleig, 1999; Ré, 2007; Rocha et al., 2015). No entanto, poucos são os trabalhos que relacionam a mirmecofauna em áreas de plantios de Pinus (Matos et al., 1994; Lutinski et al., 2008; Souza, 2010).

Neste sentido, este trabalho teve por objetivo determinar as espécies de formicídeos que ocorrem em três plantios do gênero Pinus com diferentes idades, comparando com um remanescente de floresta nativa. Além de avaliar a influência de características abióticas na amostragem de formigas.

\section{MATERIALE MÉTODOS}

O estudo foi conduzido durante o período de um ano, no município de Irati-PR (2528’02"S e 50³9’04"W) (Figura 1), localizado no segundo planalto paranaense a cerca de 836 metros de altitude em relação ao nível do mar, em quatro ambientes distintos (Tabela 1), próximos entre si, mas não adjacentes. As áreas de estudo pertenciam ao Colégio Florestal de Irati.

De acordo com Sistema de Classificação Climática de Köppen, o clima da região onde o estudo foi conduzido é do tipo Cfb - Subtropical Úmido Mesotérmico, com geadas severas e frequentes, sem estação seca (SPVS, 1996) e o solo predominante dos locais da pesquisa é classificado como Cambissolo, de acordo com o levantamento edáfico da instituição (não publicado) e confirmado por Mazza (2006).

Os dados meteorológicos - precipitação, temperatura média, temperaturas máxima e mínima e umidade relativa do ar - foram fornecidos pelo Instituto Nacional de Meteorologia (INMET), o qual possuí uma estação meteorológica localizada na área de domínio do Colégio Florestal de Irati.

A amostragem ocorreu em quatro etapas distribuídas ao longo de um ano, nos meses: maio, agosto, setembro e dezembro. As coletas dos formicídeos foram realizadas por meio de armadilhas de queda do tipo pitfall adaptadas para a utilização de iscas de sardinha (sobre o recipiente foi disposta uma prancha de madeira de 30 x $30 \mathrm{~cm}$ com $10 \mathrm{~cm}$ de altura, no centro da estrutura estava fixado um prego, onde fora amarrado um barbante contendo papel alumínio com sardinha enlatada em óleo comestível) (adaptado de Aquino et al., 2006). Os recipientes plásticos ( $500 \mathrm{~mL}$ de capacidade contendo 100 mL de uma solução com álcool a 70\% e detergente) foram enterrados verticalmente no solo, de forma que a borda superior ficasse alinhada com a superfície. As 

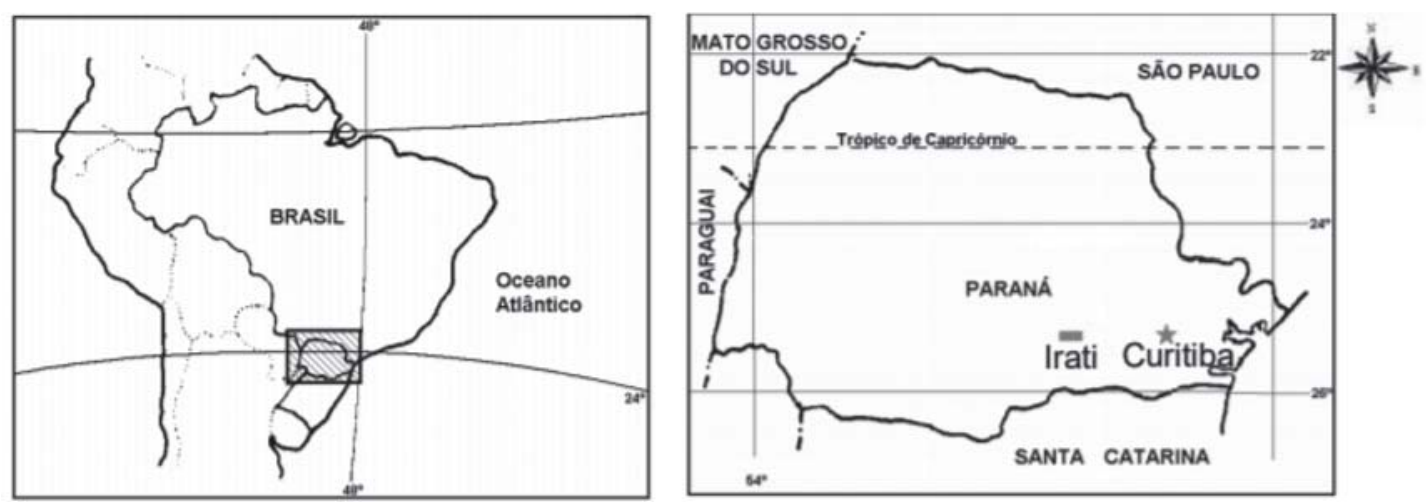

Figura 1 - Mapa de localização do município de Irati, PR.

Fonte: Galvão et al., 2002, citado por Souza (2010).

Tabela 1 - Descrição dos ambientes de estudo localizados no município de Irati, PR

\begin{tabular}{ccl}
\hline Tratamento & Dimensão do talhão & \multicolumn{1}{c}{ Descrição da área amostrada } \\
\hline P22 & 1,16 ha & $\begin{array}{l}\text { Plantio de Pinus elliottii aos } 22 \text { anos de idade com densidade de } \\
510 \text { árvores/ha. }\end{array}$ \\
P13 & 1,32 ha & $\begin{array}{l}\text { Plantio de Pinus elliottii aos } 13 \text { anos de idade com densidade de } 970 \\
\text { árvores/ha, sofreu queima parcial da área. } \\
\text { Plantio de Pinus taeda aos } 8 \text { anos de idade com densidade de } 1286 \\
\text { Ṕrvores/ha. } \\
\text { Remanescente de Floresta Ombrófila Mista, pouco antropizado, utilizado } \\
\text { principalmente para aulas práticas de ecologia, dendrologia, educação } \\
\text { ambiental e solos. }\end{array}$ \\
\hline
\end{tabular}

armadilhas foram dispostas em transectos de $50 \mathrm{~m}$, com espaçamento de $10 \mathrm{~m}$ entre elas, totalizando cinco armadilhas por área (adaptado de Fonseca \& Diehl, 2004).

No processo de triagem os indivíduos coletados foram acondicionados em frascos menores e levados ao Laboratório de Proteção Florestal da Universidade Federal do Paraná (UFPR-Curitiba/PR). Para identificação, os espécimes, foram enviados aos cuidados do pesquisador Doutor Pedro Pacheco dos Santos Lima da Universidade de São Paulo, Faculdade de Medicina Veterinária e Zootecnia, campus Pirassununga/SP.

Foram listados os números de espécies encontradas e sua ocorrência ou não nas armadilhas iscadas e no período de ocorrência (época da coleta). Para tal, foi realizado um Delineamento Fatorial com quatro áreas distintas de coleta (Fator 1) e duas épocas de coleta (Fator 2), totalizando cinco repetições em cada área. A análise estatística foi realizada no programa ASSISTAT
Versão 7.7 Beta onde se comparou as médias de ocorrência do número de indivíduos e espécies por área e época de coleta, pelo teste de Tukey a 5\% de significância.

Em seguida foi determinada a frequência relativa em porcentagem como medida de abundância das espécies (Duarte, 1993; Lima, 2000). A frequência relativa utilizada como medida de abundância das espécies (Silveira-Neto et al., 1976) foi calculada para cada ecossistema: \%f $=(n i / n t) 100$. Onde ni é a frequência absoluta (número de registros da espécie “i”) nt é a somatória do registro de todas as espécies no ecossistema (Lima, 2000). A definição de espécies consideradas como bioindicadoras ocorreu com base na literatura.

\section{RESULTADOS E DISCUSSÃO}

No total, foram coletados 3374 indivíduos da Família Formicidae distribuídos em três subfamílias, sete tribos e gêneros. Dezesseis indivíduos não foram identificados 
ao nível de Subfamília. Entre os tratamentos avaliados “Fator Área” (quatro ambientes distintos), a análise não apontou diferença significativa para as variáveis amostradas - número de indivíduos quanto para o número de espécies.

O tratamento FOM registrou o menor número de indivíduos capturados 6,94\%, tal área comparada às demais, possuí estrutura vertical mais definida, sendo compostas por diferentes estratos vegetais e apresentado maior diversidade florística. Essa estrutura pode conferir a área maior adaptação de diversas populações que tendem reduzir o número de indivíduos devido à competição. Esse fato é relatado por Silveira Neto et al. (1976), em que destacam que a competição entre duas ou mais espécies resulta na substituição de uma das espécies, ou força ambas, a viverem juntas, em um equilíbrio populacional, com densidade reduzida compartilhando as fontes de energia. De acordo com Lawton (1983), habitats complexos estruturalmente abrem oportunidade de instalação e sobrevivência de maior número de espécies decorrente do aumento da capacidade de suporte do meio, representado pela maior variedade de recursos disponíveis, alimentação e esconderijos.

Ocorreram nove espécies do gênero Pheidole (Myrmicinae: Pheidolini) (Tabela 1) distribuídos em todas as áreas de estudo, somados representam 57,11\% dos formicídeos amostrados. Esse resultado corrobora com Sousa et al. (2015) que também registraram o gênero em três ambientes distintos no Bioma Caatinga. De acordo com Hölldobler \& Wilson (1990) trata-se de um gênero com o maior número de espécies na América.

Na área de estudo FOM, entre os formicídeos amostrados o gênero Pheidole teve a maior frequência corroborando os dados de Alves (2007). Ainda neste ambiente (FOM) outro gênero que teve destaque em relação à frequência comparada aos demais ambientes, foi o gênero Pachycondyla (Ponerinae: Ponerini). Esse resultado corrobora os dados obtidos por Ilha et al. (2009) que registraram o gênero Pachycondyla em todas as áreas de estudo (Eucalyptus grandis, Floresta Ombrofila Mista e banhado), sendo mais frequente na área de mata nativa. O gênero Pachycondyla é agrupado com espécies de formigas predadoras (Delabie et al., 2000) além de apresentarem espécies generalistas e também especialistas, que constroem seus ninhos no solo, em madeira em condições de apodrecimento (Lattke, 2003) associadas às plantas ou epífitas (Brandão et al., 2009). Essas condições são encontradas no local de estudo (FOM), onde, este gênero teve a maior frequência. Estudando ambientes degradados pela mineração de diamante em Poraxéu (MT), Rocha et al., 2015 concluíram que uma espécie deste gênero, Pachycondila crassinoda pode ser bioindicadora de ambientes preservados e estabilizados ecologicamente.

Ainda com relação ao gênero Pachycondila, os resultados obtidos também corroboram com Cantarelli et al. (2015) que comparou quatro ambientes no município de Frederico Westphalen - RS e registrou a maior incidência do gênero na Floresta Nativa (ambiente mais conservado).

Nas áreas P22 e P13, o gênero Pheidole também apresentou maior frequência, quando comparado com os demais gêneros presentes em cada área (Tabela 1) tal resultado também foi observado por Cantarelli et al. (2015). Essa amostragem reforça a característica de ser naturalmente abundante, como cita Alves (2007) além de apresentarem espécies generalistas (Andersen, 2000).

A área P8 registrou o maior número de indivíduos das espécies do gênero Solenopsis (Myrmicinae: Solenopsidini) (Tabela 2 ), tal resultado diverge de Cantarelli et al. (2015). Os autores registraram o gênero em quatro ambientes estudados (Floresta nativa, Eucalipto, Agricultura e Pastagem exótica) apesar do gênero ter sido registrado nos quatro ambientes a maior abundância foi observada na Floresta nativa.

O gênero Solenopsis é onívoro de serapilheira e detritívoro (Delabie et al., 2000). Aárea onde ocorreu a predominância de Solenopsis, tratava-se de um plantio de Pinus taeda aos oito anos de idade que sofreu incêndio superficial e no momento do estudo não apresentava sub-bosque. Essas características corroboram com Marinho et al. (2002) que relatam que espécies desse gênero apresentam agressividade quanto à utilização dos recursos, e, podem suportar períodos de escassez de alimento (Marinho et al, 2002).

A espécie Camponotus rufipes (Formicinae: Camponotini) teve maior frequência na área de Pinus sp. aos 13 anos de idade, espécies do gênero Camponotus são onívoras verdadeiras (Delabie et al., 2000) e a espécie Camponotus rufipes é característica de locais abertos. Cabe destacar que na ocasião do estudo esta área apresentava 970 arvores/ha sem presença 
Tabela 2 - Frequência relativa das espécies de formicídeos edáficos encontrados em quatro ambientes no Paraná, 2017

\begin{tabular}{|c|c|c|c|c|}
\hline \multirow{2}{*}{ Espécies } & \multicolumn{4}{|c|}{ Frequência relativa (\%) } \\
\hline & FOM & $\mathrm{P} 22$ & $\mathrm{P} 13$ & P 8 \\
\hline \multicolumn{5}{|l|}{ Subfamília Formicinae } \\
\hline Camponotus rufipes & & 0,93 & 8,79 & 0,38 \\
\hline Camponotus sp. 1 & 5,55 & 0,39 & 0 & 1,81 \\
\hline \multicolumn{4}{|l|}{ Tribo Lasiini } & 0,47 \\
\hline Paratrechina sp. 1 & 0,42 & 0,13 & 0,14 & 0,76 \\
\hline Paratrechina sp. 2 & 0,42 & 1,99 & 0,07 & 4,21 \\
\hline Paratrechina sp. 3 & 0 & 0 & 0 & 0,38 \\
\hline \multicolumn{5}{|l|}{ Tribo Não Identificada } \\
\hline Espécie 1 & 0,42 & 30,05 & 0,29 & 2,00 \\
\hline \multicolumn{5}{|l|}{ Subfamília Myrmicinae } \\
\hline Acromyrmex subterrabeus subterraneus & 0 & 0 & 0 & 0,47 \\
\hline Acromyrmex subterrabeus & 0 & 7,18 & 0 & 0 \\
\hline Acromyrmex ambiguus & 0 & 0,66 & 0 & 0 \\
\hline \multicolumn{5}{|l|}{ Tribo Myrmicini } \\
\hline Pheidole sp. 1 & 0 & 0 & 1,26 & 0 \\
\hline Pheidole sp. 2 & 0,42 & 0 & 0,14 & 0 \\
\hline Pheidole sp. 3 & 37,6 & 55,05 & 26,28 & 3,34 \\
\hline Pheidole sp. 4 & 0 & 0 & 0 & 0 \\
\hline Pheidole sp. 5 & 0 & 0 & 50,03 & 0 \\
\hline Pheidole sp. 6 & 0,42 & 0 & 4,31 & 0,095 \\
\hline Pheidole sp. 7 & 14,96 & 0 & 3,57 & 0 \\
\hline Pheidole sp.8 & 13,24 & 0,14 & 0 & 4,01 \\
\hline Pheidole (gr. fallax) & 8,97 & 0,39 & 3,49 & 5,45 \\
\hline \multicolumn{5}{|l|}{ Tribo Solenopsidini } \\
\hline Solenopsis sp. & 0 & 0 & 0,14 & 74,35 \\
\hline \multicolumn{5}{|l|}{ Subfamília Ponerinae } \\
\hline \multicolumn{5}{|l|}{ Tribo Ponerini } \\
\hline Pachycondyla sp. & 16,24 & 1,46 & 0,74 & 2,20 \\
\hline Subfamília não Identificada & 0 & 0 & 0 & 0 \\
\hline Espécie 2 & 0 & 0 & 0,67 & 0 \\
\hline Espécie 3 & 0,85 & 0,53 & 0 & 0 \\
\hline Espécie 4 & 0,42 & 0 & 0 & 0 \\
\hline
\end{tabular}

de sub-bosque. Rocha et al. (2015) destaca que uma espécie de Camponotus encontrada em seu trabalho pode ser considerada como bioindicadora da degradação ambiental ocasionada pela mineração de diamante.

O gênero Paratrechina ocorreu em todos os ambientes com maior frequência na área P8 (Tabela 1), Matos et al. (1994) também registrou tal gênero em área de Pinus e Cantarelli et al. (2015) também registrou o gênero nos ambientes estudados tendo a maior frequência em área de pastagem exótica. Trata-se de um gênero oportunista e caracterizado por formigas não especialistas, pouco competitivas e que possuem ampla distribuição, predominando em locais com altos índices de estresse e distúrbio (Andersen, 2000; Fernandéz, 2003).

As épocas de coleta (maio, agosto, setembro e dezembro) estão apresentadas na Tabela 3. De acordo com Kaspari (2000) os fatores abióticos como temperatura e umidade, podem regular o acesso dos formicídeos aos recursos (alimento e lugares para nidificação). $\mathrm{O}$ maior número de espécies coletado foi no mês de dezembro que apresenta diferença estatística quando 
comparado as demais coletas. Os meses setembro e agosto tiveram as menores frequências de coleta (espécie e indivíduos), o mês de setembro coincidiu com o maior índice pluviométrico, 369,2 mm (Figura 1).

A influência da precipitação sobre o número de indivíduos coletados ficou caracterizada na coleta setembro, alguns dias que antecederam a coleta foram registrados altos índices de precipitação (Figura 2). Este resultado corrobora os dados apresentados por Della-Lucia (1982) que estudando ordenação de formicídeos em quatro agroecossistemas na cidade de Viçosa-MG, também verificou declínio na abundância de formicídeos após um período de precipitação. Kaspari (2000) também relata que a abundância de água na vegetação diminui as atividades das formigas, em especial as formigas de pequeno porte.

Os dados fornecidos pelo Instituto Nacional de Meteorologia (INMET) mostraram que a menor temperatura média foi registrada no mês de junho enquanto que novembro registrou as maiores temperaturas do ano (Figura 2). As maiores frequências de

\section{CONCLUSÃO}

O gênero Solenopsis foi o mais frequente no plantio de Pinus aos oito anos de idade, revelando que o gênero pode estar associado a ambientes perturbados enquanto que o gênero Pachycondyla foi observado com maior frequência em ambiente com melhor estado de conservação. A temperatura e a precipitação influenciaram a coleta de formicídeos. O aumento da precipitação reduziu o número de indivíduos coletados.

\section{LITERATURACITADA}

\section{ASSIS, F. Assistat - Assistência}

Estatística. Versão 7.7. Beta 2014. In: http:// www.assistat.com/indexp.html

Tabela 3 - Formicídeos edáficos coletados em diferentes épocas ao longo de um ano no município de Irati, PR

\begin{tabular}{lcccc}
\hline \multirow{2}{*}{ Variáveis } & \multicolumn{4}{c}{ Épocas de coleta } \\
\cline { 2 - 5 } & Maio & Agosto & Setembro & Dezembro \\
\hline Número de espécies & $2,45 \mathrm{~b}^{*}$ & $1,00 \mathrm{c}$ & $1,55 \mathrm{bc}$ & 4,15 a \\
Número de indivíduos & 45,20 ab & $6,45 \mathrm{~b}$ & $3,90 \mathrm{~b}$ & 114,85 a \\
\hline
\end{tabular}

* As médias seguidas pela mesma letra não diferem estatisticamente entre si. Foi aplicado o Teste de Tukey ao nível de 5\% de probabilidade.

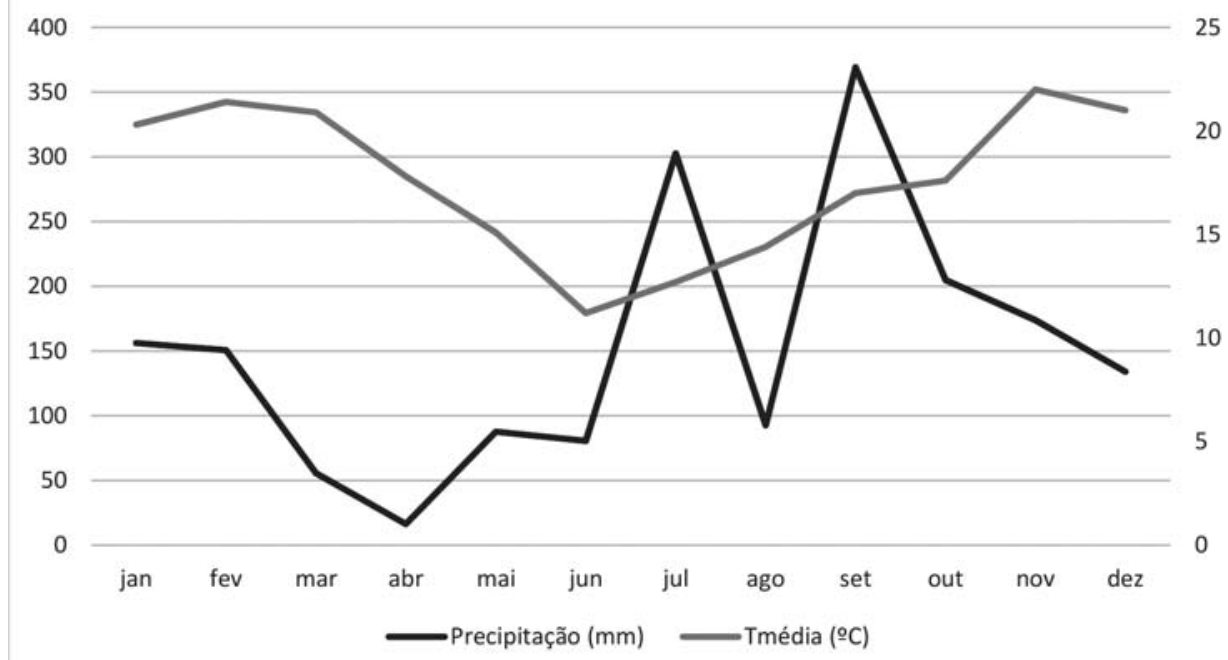

Figura 2 - Precipitação média mensal (mm) e temperatura média (em graus Celsius) ao longo do ano 2009 no município de Irati-PR.

Fonte: Instituto Nacional de Meteorologia (INMET) (2009). 
ALONSO, L.E.; AGOSTI, D. Biodiversity studies, monitoring, and ants: an overview. In: AGOSTI, D.; MAJER, J.D.; ALONSO, L.E. et al. Ants standard methods for measuring and monitoring biodiversity. 2000. p.01-08.

ALVES, H.S.R. Identificação de bioindicadores e planejamento de mini-corredores ecológicos na área de proteção ambiental Costa de Itacaré/ Serra Grande, Bahia. 113f. Dissertação de Mestrado. Programa Regional de Pós-graduação em Desenvolvimento e Meio Ambiente. Mestrado em Desenvolvimento Regional e Meio Ambiente. Universidade Estadual de Santa Cruz. Ilhéus, Bahia, 2007.

ANDERSEN, A.N. A global ecology of rainforest ants: Functional groups in relation to environmental stress and disturbance. In: AGOSTI, D.; MAJER, J.D.; ALONSO, L.E.; SCHULTZ, T.R. Ants standard methods for measuring and monitoring biodiversity. 2000, p.25-34.

BRANDÃO, R.F.; SILVA, R.R.; DELABIE, J.H. Formigas (Hymenoptera). In: PANIZZI, A.R.; PARRA, J.R.P. (Ed.) Bioecologia e nutrição de insetos - Base para o manejo integrado de pragas. Brasília, DF: EMBRAPA Informação Tecnológica, capítulo 9, p.323-369, 2009.

BORROR, D.J.; DELONG, D.M. Introdução ao estudo dos insetos. São Paulo: Edgar Bluncher Ltda, 1969.

DELABIE, J.H.C.; AGOSTI, D.; NASCIMENTO, I.C. Litter ant communities of the Brasilian Atlantic rain forest region. In: AGOSTI, D.; MAJER, J.D.; SCHULTZ, T. (Ed.) Sampling grounddwelling ants: Case studies from the World's rain forests, 2000. p.1-17.

CANTARELLI, E.B.; FLECK, M.D.; GRANZOTTO, F. et al. Diversidade de formigas (Hymenoptera: Formicidade) da serapilheira em diferentes sistemas de uso do solo. Ciência Florestal, Santa Maria, v.25, n.3, p.607-616, jul.-set., 2015.

DELLA LUCIA, T.M.C.; LOUREIRO, M.C.; CHANDLER, L. et al. Ordenação de comunidades de Formicidae em quatro agroecossistemas em Viçosa, Minas Gerais. Experientiae, Viçosa, v.28, n.6, p.68-94, 1982.
DIAS, N.S. Interações entre as comunidades de formigas (Hymenoptera: Formicidae) de fragmentos florestais e de agroecossistemas adjacentes. Dissertação de Mestrado. Universidade Federal de Lavras UFLA, 2004.

DIEHL-FLEIG, E.; LUCCHESE, M.E.P.; SANHUDO, C.E. et al. Mirmecofauna de solo em áreas de mineração de cobre na bacia do Camaquã, RS, Brasil. Naturalia, São Paulo, v.24 (n. esp.), p.99-101, 1999.

DUARTE, L.C. Guildas em formigas epigeicas (Hymenoptera: Formicidae) em uma área de Mata Atlântica. 69f. Dissertação de Mestrado. Instituto de Biociências do campus de Rio Claro, Universidade Estadual Paulista. Rio Claro, 1993.

FERNÁNDEZ, F. Breve introduccion a la biologia social de las hormigas. In: FERNÁNDEZ, F.

Introducción a las hormigas de la region Neotropical. Bogotá: Instituto de Investigación de Recursos Biológicos Alexander von Humboldt, capítulo 5, p.89-96, 2003.

FREITAS, A.L.V.; FRANCINI, R.B.; BROWN Jr., K.S. Insetos como indicadores ambientais. In: CULLEN Jr., L.; RUDRAN, R.; VALLADARESPADUA, C. (org.) Métodos de estudos em biologia da conservação e manejo da vida silvestre. 2 ed. Curitiba: UFPR, 2009. p.125-151.

FONSECA, R.C.; DIEHL, E. Riqueza de formigas (Hymenoptera, Formicidae) epigéicas em povoamentos de Eucalyptus spp. (Myrtaceae) de diferentes idades no Rio Grande do Sul, Brasil.

Revista Brasileira de Entomologia, v.48, n.1, p.95-100, março/2004.

HÖLLDOBLER, B.; WILSON, E.O. The ants. Cambridge: The Belknap Press of Harvard University Press, 1990. p.731-732.

KASPARI, M.A. Primer on ant ecology. In: AGOSTI, D.; MAJER, J.D.; ALONSO, L.E; SCHULTZ, T.R. Ants standard methods for measuring and monitoring biodiversity, chapter 2, p.9-24, 2000. 
LATTKE, J.E. Subfamília Ponerinae. In: FERNÁNDEZ, F. (Ed.) Indroducción a las hormigas de la region neotropical. Bogotá: Instituto de Investigación de Recursos Biológicos Alexander von Humboldt, 2003. p.261376.

LAWTON, J.H. Plant architeture and diversity of phytophagous insects. Annual Review Entomology, Palo Alto, n.28, p.23-39, 1983.

LIBERAL, C.N.; GOMES, J.P.; BARRETO, T.M.S.P. et al. Riqueza de formigas (Hymenoptera: Formicidae) em um fragmento de Mata Atlântica de Pernambuco, Brasil. Anais

do VIII Congresso de Ecologia do Brasil, 23 a 28 de setembro de 2007, Caxambu MG. 2p.

LIMA, P.P.S. Impacto de formicida granulado na mirmecofauna de ecossistemas florestais. $75 \mathrm{f}$. Tese de Doutorado. Programa de Pós-graduação em Ecologia e Recursos Naturais, do Centro de Ciências Biológicas e da Saúde da Universidade Federal de São Carlos. São Carlos, 2000.

LUTINSKI, J.A.; GARCIA, F.R.M.; LUTINSKI, C.J. et al. Diversidade de formigas na Floresta Nacional de Chapecó, Santa Catarina, Brasil. Ciência Rural, Santa Maria, v.38, n.7, p.1810-1816, out/2008.

MAJER, J.D. Ants: bioindicators of Minesite Rehabilitation, land use, and land conservation. Enviromental Managment, New York, v.7, p.375-383, 1983.

MAJER, J.D.; NICHOLS, O.G. Long-term recolonization patterns of ants in Western Australian rehabilitated bauxite mines with reference to their use as indicators of restoration success. Journal of Applied Ecology, v.35, p.161-182, 1998.

MARINHO, C.G.S.; ZANETTI, R.; DELABIE, J.H.C. et al. Diversidade de formigas (Hymenoptera: Formicidae) da serrapilheira em eucaliptais (Myrtaceae) em área de cerrado em Minas Gerais. Neotropical Entomology, v.31, n.2, p.187-195, 2002.
MATOS, J.Z.; YAMANAKA, C.N.; LOPES, B.C. et al. Comparação da fauna de formigas em áreas de plantio de Pinnus elliotii, com diferentes graus de complexidade estrutural, em Florianópolis/SC. Biotemas (UFSC), Florianópolis, SC, v.7, n.1, p.57-64, 1994.

MAZZA, C.A.S. Caracterização ambiental da paisagem da microrregião colonial de Irati e zoneamento ambiental da floresta nacional de Irati. Tese de Doutorado. Programa de Pós Graduação em Ecologia e Recursos Naturais. Centro de Ciências Biológicas e da Saúde - Universidade Federal de São Carlos, 2006. 147p. São Carlos: UFSCAR.

MEADRO, M.J. Apresentação. In: SHIMIZU, J.Y. (Ed.) Pinus na Silvicultura Brasileira. Colombo: Embrapa Florestas, 2008. 223p.

OLIVEIRA, M.A.; GOMES, C.F.F.; PIRES, E.M. et al. Bioindicadores ambientais: insetos como um instrumento desta avaliação. Revista Ceres, Viçosa, v.61, Suplemento, p.800-807, nov/dez, 2014.

OLIVEIRA, I.R.; FERREIRA, N.A.; VIANA JUNIOR, A.B. et al. Diversidade de formigas (Hymenoptera: Formicidae) edáficas em três estágios sucessionais de mata atlântica em São Cristóvão, Sergipe. Agroforestalis News, v.1, n.1, 2016.

ROCHA, W.O.; DORVAL, A.; PERES FILHO, O. et al. Formigas (Hymenoptera: Formicidae) bioindicadoras de degradação ambiental em Poxoréu, Mato Grosso, Brasil. Floresta e Ambiente, v.22, n.1, p.88-98, 2015.

SILVA, A.F.; CARVALHO, Y.C.; COSTA, S.J.M. et al. Fauna de Formigas (Hymenoptera, Formicidae) em um fragmento de Floresta Atlântica no Estado de Minas Gerais. Revista Brasileira de Zoociências, v.19, n.1, p.44-55, 2018.

SILVEIRA NETO, S.; NAKANO, O.; BARBIN, D. et al. Manual de ecologia dos insetos. São Paulo: Agronômica Ceres, 1976. 419p.

SOUSA, I.D.; MARINHO, C.G.S.; LIMA, A.S. et al. Diversidade de formigas (Hymenoptera: Formicidae) epigéias na mesoregião do sertão paraibano. Revista Brasileira de Zoociências, v.16, p.43-53, 2014/2015. 
SOUZA, K.K.F. Diversidade de formigas (Hymenoptera: Formicidae) epigéicas em áreas de plantios de Pinus sp., Mata Nativa e pastagem. Dissertação de Mestrado. Programa de Pós-graduação em Engenharia Florestal. Universidade Federal do Paraná. Curitiba, 2010.

SPOLIDORO, M.V. Levantamento da mirmecofauna de solo (Hymenoptera: Formicidae) em cultivo orgânico de café (Coffea arabica). 73f. Dissertação de Mestrado. Programa de Pós-graduação em Ciências. Escola Superior de Agricultura "Luiz de Queiroz”, Piracicaba, 2009.
SPVS. Sociedade de Pesquisa em Vida Selvagem e Educação Ambiental. Nossas Árvores: Manual para recuperação da reserva florestal legal. Curitiba: FNMA, 1996. 84p.

RÉ, T.M. O Uso de formigas como bioindicadores no monitoramento ambiental de revegetação de áreas mineradas. 244f. Tese de Doutorado. Escola Politécnica da Universidade de São Paulo.

VASCONCELOS, H.L. Respostas das formigas à fragmentação florestal. Série Técnica IPEF, v.12, n.32, p.95-98, dezembro/1998.

Recebido para publicação em 6/7/2017 e aprovado em 8/2/2018. 